\title{
A Case Study on Maladjustment of Interpersonal Relationship in Freshmen
}

\author{
Lan Xingping, Yang Tao, Chen Guoqin, Sun Yun
}

Yunnan Jiaotong College, Kunming, Yunnan,650500

Keywords: General Psychological Problems, Reasonable Emotional Therapy, Case Study

\begin{abstract}
This paper studies a case of anxiety counseling in which college students suffer from maladjustment of dormitory interpersonal relationship. Zhang, an 18-year-old female freshman, because of unfriendly roommates, felt "hostile", distressed, and anxious. Thus, consultants mainly use reasonable emotional therapy to help her find out root causes of psychological problems, change the unreasonable faith in interpersonal communication. After counseling for four times, ultimately, the inner distress and anxiety is addressed, and the counseling has achieved the desired effect.
\end{abstract}

\section{General Information}

(1) General situation:

Zhang, female, Han nationality, 18 years old, freshman, Yunnan native, emaciated, face haggard, from the rural market town, parents are working at home, a small three-year-old brother, the family economy in general.

(2) Personal history of growth:

Living in rural market town, grew smart and studious, did not get serious illness, but the body is thin. He has no prosecution family genetic disease, history of mental illness. From primary to high school, learning has been very hard, due to over-emphasis on learning, not good at communicating with others, not accustomed to collective life, after entering college, tension with the hostess in January.

(3) Appearance and behavior:

Dress neat, face-length commensurate, thin body, looking pale. Look nervous, anxious. Expression and language expression coordination, the sound is not big, some hesitate, but the train of thought is clear.

(4) Inspiration and judgment:

There is a strong desire for help, can be aware of their emotions, emotional problems, more accurate on their own judgments, there is a more clear purpose of consultation.

(5) Social function:

When talking to people nervous anxiety, class concentration can not be focused, but she also tries to study hard, academic performance at a medium level. No love.

(6) The first psychological test results:

To do SCL-90 self-rating scale, the results show: interpersonal sensitivity 2.5, anxiety 2.4, can be seen from the first test, Zhang interpersonal relationships sensitive factor scores high, followed by anxiety factor scores high.

\section{The Main Statement and Personal Statement}

(1) Chief Complaint:

In the past January, with anxiety and irritability, I felt depressed in returning to my dorm room. I felt that I did not like myself and hated myself, so I did not talk to my roommate either. One Counselor had requested to change dormitory, but did not get consent.

(2) Personal statement:

Teacher, can you help me find a solution, let me change dormitory? When I got back to the dormitory, I was baffled because I quarreled with a little bit. In this dormitory, I feel lonely and 
painful, and now after class I do not want to return to this dormitory, I told the counselor to change quarters, but the counselor does not agree. I often go to school now, can not concentrate on listening, sleep at night is not practical, mood irritability, learning efficiency.

\section{Observe and Understand the Situation}

The solicitor came alone, neatly dressed, polite, and nervous about the dorm. Clear thinking, good knowledge, there is a desire to seek treatment.

According to the helper who looked for seekers, those who seek help living habits are not good, shoes, socks disorderly placement, bed mess, often affect the performance of dormitory health assessment; seldom stay in the dormitory, seekers, are generally turned off and then returned to dormitory to sleep. The movement of its wash action is too large, seriously affecting the rest of other students, one night a roommate was awakened and said a few words, for which two people had a quarrel.

\section{Assessment and Diagnosis}

(1) Assessment

According to the collection of clinical data, the current mentality of help seekers, physical and social status as follows:

Mental state: attention easily dispersed, anxious mood, mild depression

Physical status: According to a recent physical examination report showed no physical abnormalities, but very light sleep.

Social status: social problems, interpersonal tensions

(2) Diagnosis

Comprehensive clinical data, the initial diagnosis of those who seek help is: General psychological problems.

(3) Diagnosis based on the following:

Senor emotional problems are caused by real-life events; psychological problems lasting less than a month; bad emotions can still be under the control of the mind and there is no generalization.

(4) Differential diagnosis

Differentiation from psychosis: According to the three principles of distinguishing between normal and abnormal psychology, the caller has the subjective and objective world unification, the inner coordination and consistency of mental activity, the relatively stable personality, the self-knowledge, the active seeking for help, the chaos without logic thinking, no Hallucinations, delusions and other psychiatric symptoms, excluding psychiatric problems.

Differentiated from neurosis: According to Professor $\mathrm{Xu}$ Yinshen neurosis rating criteria: less than 3 months duration of disease as short-range, rating 1; help themselves to come to counseling, seeking to get rid of, in the spirit of the degree of mild pain are mild patients, rated 1 point; social function, seeking help to work and study and interpersonal relationships only minor disturbances, rating 1 point. The total score of 3 points, can not be diagnosed as neurosis.

Serious psychological problems are identified: Serious psychological problems, strong stimulation, intense reaction and generalization, the duration of more than 2 months, have a serious impact on social function. However, the psychological problems of the help-seeking person were caused by the fact that they did not adapt to the life of the dormitory and had anxiety caused by false cognition. The reaction intensity was not so long. The duration of the symptom was not overdue and the social function was not significantly impaired, so you can rule out serious psychological problems.

(5) Etiological analysis

Physiological factors: women seeking help, 18 years old, freshman.

Social factors: no experience of living in dormitories, lack of experience in interpersonal relationships; few friends, lack of effective social support system.

Psychological factors: there is a misconception: roommates do not like themselves, they all hate 
themselves.

Personality characteristics: the pursuit of perfection

\section{Consulting Goals}

Based on the above assessment and diagnosis, consult with the help seekers to determine the following consultation objectives:

(1) Short-term goals:

To change the unreasonable conviction of those who seek help to eliminate or reduce the negative emotions of those seeking help.

(2) Long-term goal:

On the basis of meeting the above goals, the ultimate goal is to promote the mental health and personality of those seeking help.

\section{Consultation Program}

Use reasonable emotional therapy for counseling. The basic principle is: people's emotional distress is not an external event, but people's attitude, opinion, evaluation and other cognitive content, so to change the emotional distress is not committed to changing the external events, but should change the cognitive. Ellis believes that the induced event is A, people's perception is B, the individual's emotional and behavioral results of $\mathrm{C}$, so its core theory, also known as "ABC theory". [2] Change Zhang's bad cognition of interpersonal communication, so as to change his view on the roommate and improve the interpersonal relationship.

A printed copy of the written materials on the rights and obligations of both parties will be submitted to the sponsor. At the same time, applicants are advised to ask any questions they may have and to state the principle of confidentiality of the consultation.

The responsibilities, rights and obligations of both parties:

(1) The helper's responsibility:

(1) to provide counselors with psychological information related to the true information;

(2) actively and consultants to explore ways to solve the problem;

(3) completed the work agreed by both parties.

(2) Seeker's rights:

(1) have the right to know the consultant training background and qualifications;

(2) have the right to know the specific methods of consultation, the process and principles;

(3) the right to choose or replace the appropriate consultant;

(4) have the right to refer to or terminate the consultation;

(5) on the content of the consultation program have the right to information, consultation and the right to choose.

(3) Obligations of help seekers:

(1)comply with the relevant provisions of the advisory body;

(2)comply with and implement the agreed content of all aspects of the consultation program;

(3)respect consultants, observe the appointment time, if there are special circumstances to inform the consultant in advance.

(4) Consultant's responsibility:

(1)comply with professional ethics and abide by relevant state laws and regulations;

(2)help those who seek help to solve psychological problems;

(3)strict compliance with the principle of confidentiality, and explain the exception of secrecy.

(5) Consultant's Rights:

(1) have the right to know and ask for help on the psychological problems related to personal information;

(2)the right to choose the right person for help; 
(3)in the attitude of responsible person, have the right to refer to or terminate the consultation.

(6) Consultant's obligations:

(1) to help those who introduce their own training background, showing business license and qualification certificates and other related documents;

(2)comply with the relevant provisions of the advisory body;

(3)comply with and implement agreed aspects of the consultation program;

(4)respect for those who seek help, comply with the appointment time, if there are special circumstances to inform those who seek help in advance.

(7) Consultation time: once a week, every 50 minutes.

(8) Consultation Fee: According to the school rules, free consultation for students.

\section{Consultation Process}

(1) Psychological counseling stage

Diagnostic phase; comprehension phase; repair pass stage; re-education stage

(2) Specific consultation stage

(1)The first stage: the diagnostic phase

Objectives: To understand the basic situation of those seeking help; to establish a good relationship between counseling; to determine the main problems

Process: Fill in the consultation registration form, introduce the relevant matters and rules in the consultation; conduct psychological tests on the help-seekers, understand the growth process and the present conditions of life to the help-seekers, feedback the test results and determine the consultation objectives, explain the principle of confidentiality to the help-seekers and clarify. The responsibilities, rights and obligations of both parties; explain to the help-seeking theory of rational emotion therapy, and based on the $\mathrm{ABC}$ theory to analyze and diagnose the problem of the help-seeking people, and find out the concrete manifestation of his emotional distress and discomfort.Talk to your hostess; evoked events that correspond to these responses A: Quarrel with your hostess; unreasonable beliefs between the two B: I think all five roommates do not like themselves and hate themselves. Explain the theory of $\mathrm{ABC}$ to the help seeker, make her realize the relation between $\mathrm{A}, \mathrm{B}, \mathrm{C}$ and carry on the preliminary analysis according to her own question.

(2) The second stage: comprehension phase

Purpose: To further deepen the consultation relationship; to find and confirm the unreasonable conviction of those who seek help.

Process: The counselor went deeper into finding and confirming the caller's unreasonable conviction that "they both dislike me and dislike me," helping the caller to further understand that her emotional problems were caused by the irrational beliefs he now holds. She is responsible for her. Only changing unreasonable beliefs can change emotional distress.

The following is a consultation piece:

Zhang: Teacher, I was so hurt when I quarreled that night ,so that I grew more and more arrogant. I have been let them everywhere, and even in the dormitory, and they still deliberately find me embarrassed. I think only for the dormitory can solve my problem.

Counseling: Why did you change your dormitory?

Zhang: Because of the people in this dormitory they hate me, do not like me; other quarters do not hate me.

Counseling: Can you make sure you've got a new one, do your neighbors love you?

Zhang: (silence moment) is not necessarily.

Counseling: That is to say for the new quarters, your relationship with the new hostess is good or bad is not necessarily?

Zhang: Well.

Counseling: Now the dorm roommates may not hate you, do not like you, but they do not like what you do? The other person happens to do exactly what they do not like, and they are equally attuned to the other person. They are not targeting something, not someone. 
Zhang: (silence)

I think they do not like a few things, you are over-generalized as you do not like this person.

Zhang: There is also this possibility.

Consultation: After you go back and think about it, how do you want your roommate to treat you? Write it down; how you treat your roommate yourself, then write it down. And then compare to see if the actual situation of your own housemate treats you as you would expect.

(3)The third stage: repair phase

Objective: To employ multiple techniques to enable callers to amend or abandon their original irrational beliefs and replace them with reasonable beliefs so as to reduce or eliminate emotional symptoms.

Process: In the process of combing homework, by contrast with the seeker treat himself and his roommate hostess, let them understand that they have absolute requirements for others or the surrounding environment; in the process, the combination of behavioral therapy method; counseling teach assistants to imagine relaxation exercises, to relax in the dormitory, and to introduce the principles and methods of interpersonal relationships, such as empathy, "do not give up on others,".

Help those who seek help to improve their interpersonal skills; homework assignments: ask those who seek assistance in the use of methods in their daily lives try to engage in useful exchanges with the hostess. The following is a consultation piece:

Zhang: Teacher, I wrote what you taught us last time.

Consultation: done quite well, after writing what new discovery?

Zhang: (Read carefully what you wrote.) According to the writing, I found myself wanting to treat myself as a hostess, but I could not do it for my roommate.

Consultation: Can you give an example to explain?

Zhang: I hope I will not be disturbed when I go to bed, but other people go to sleep at night time is just my time back to the dorm, I have no other solution, had to disturb them.

I think they mainly do not like to be disturbed when they sleep, not to hate someone.

Zhang: It should be.

Consultation: What time do you go back to dormitory at night?

Zhang: Turn off the lights, it is estimated about 11:30 or so.

Counseling: What are you doing before you go back to your dorm? Working outside school?

Zhang: No, go to the dormitory next door to find a chat to come to the fellow.

Consultation: It can adjust their schedule, fight back to dormitory at 10:30 to wash, go out to sleep on time 11:00 lights?

Zhang: I try to adjust it.

Counseling: Since you want to sleep without being disturbed by others, when others go to sleep, they should be lighter regardless of what they do, try not to disturb others.

Zhang: This one I can do.

Advisor: Your health score high quarters?

Zhang: Not high.

Advisory: The main reason for being deducted points it?

Zhang: Shoes and socks are not placed neatly, the bed quilt is not folded.

Advisory: You manage yourself first, before each health check their shoes and socks placed well, the quilt folded, slowly, we all do, and your quarters score will be high.

Zhang: Well, start from yourself.

Counseling: You can ask your roommate, before what their own behavior disturbed them, whether they have any good advice on similar disturbances?

Zhang: Let me try it.

(4)The fourth stage: Re-education stage

Objective: To consolidate the effects of treatment in the first few stages so that callers can still use what they have learned to deal with problems in their lives after the counseling is concluded; and to end the counseling.

Process: Make help-seeking people aware of their own anxiety, irritability and other emotions is 
their own irrational conviction; consultants still use the methods and techniques mentioned in the previous stage to help those seeking help in the cognitive approach, thinking process and emotional and behavioral performance and other aspects of the establishment of a new response model; the use of psychological tests to re-evaluate the counselor after the emotional experience and behavior; end of the consultation.

\section{The Advisory Effect Evaluation}

(1) Self-assessment for help seekers: Through counseling, symptoms have been greatly improved. And friends of hostels get along well, do not change the dormitory, the mood has returned to normal, thanks to the guidance of the counselor, satisfied with the results of the consultation.

(2) Recruiter roommate assessment: distress anxiety disappeared, and the exchange of homes interaction back to normal.

(3) Consultant evaluation: After seeking help, the mood is obviously better than just consulting. Telephone follow-up after a month, said the advisory effect continues to be maintained, the symptoms basically eliminated.

(4) Consultation test results: SCL-90, a total score of 151 points, anxiety 1.4 points, interpersonal 1.6.

\section{Conclusions}

All in all, by seeking help from the consultants, students who have psychological problems can overcome the inner distress and anxiety. Consultants mainly use reasonable emotional therapy to help those who seek analysis of the root causes of psychological problems, change the unreasonable faith in interpersonal communication, and ultimate achieve the desired effect.

\section{Acknowledgements}

This article is the phased achievement of "Innovative Action Plan Project of Yunnan Jiaotong College"Carrying Out the Standards of Professional Ability of College Counselors (Interim)" (Proje ct No. RW-59).

\section{References}

[1] Counseling counseling tutorial writing group. Psychological counselors three (basic knowledge) [M]. 2017,2: 96-98. Beijing: Business Management Press.

[2] Counseling counseling tutorial writing group. Psychological counselors three (skills operation) [M]. 2017,2: 52-58. Beijing: Business Management Press.

[3] Counseling counseling tutorial writing group. Psychological counselor two (basic knowledge) [M]. 2016,9: 2-5. Beijing: Business Management Press.

[4] Counseling counseling tutorial writing group. Psychological counselor two (skills operation) [M]. 2016,9: 98-99. Beijing: Business Management Press. 